\title{
Robust and Efficient Driver Monitoring System Using a Synthesis of Facial Features and Biosignals
}

\author{
S.P. Predeep Kumar and E. Babu Raj \\ ${ }^{1}$ Research Scholar, St.Peter's University, Chennai, Tamilnadu, India. \\ ${ }^{2}$ Principal, N S College of Engineering, Kanayakumari Dist., Tamilnadu, India
}

DOI: http://dx.doi.org/10.13005/bbra/1969

(Received: 19 June 2015; accepted: 11 August 2015)

\begin{abstract}
Monitoring the driver's state of consciousness and fatigue is exclusively important to reduce the number of traffic accidents. We have proposed a collective data fusion method for checking driver safety levels by combining eye features and Heart rate Variability (HRV). Fatigue behavior was determined via facial image processing using Matlab as a tool. After detecting the face, the location of the eyes will be detected using the duration of eye closure, and the frequency of eye blinks. It is used as a distinctive characteristic to judge whether a driver is drowsy or not. General duration of closure is 0.15 to 0.25 seconds. Initially the HRV analysis was accomplished by acquiring Electrocardiogram (ECG) signal through non-intrusive ECG sensors wrapped on to the steering wheel followed by filtering noises and we have calculated pulse rate via peak detection using LabVIEW. If there are any abnormal signs found in the ECG of the driving person then the patient is wide-open to have a second order attack. The corresponding heart rate is sent as an alert SMS to the medical practitioner by microcontroller through GSM modem.
\end{abstract}

Key words: Biomedical signal processing, Electrocardiogram, Fatigue, Image processing, Noise Shaping, Signal processing algorithms.

Driver fatigue and heart attacks at road are the remarkable factors for a huge number of vehicle accidents. Driver Fitness and Monitoring is responsible for reviewing driving privileges of individuals and assessing their ability to safely operate motor vehicles. Some of the diseases or disabilities that may interfere with the safe operation of vehicles can be found in these categories: Vision (e.g. minimum correct vision, double vision, telescopic lenses), Hearing (e.g. vertigo, issues particular to commercial drivers), Cardiovascular diseases (e.g. heart disease, heart attack), Cerebrovascular Diseases (e.g. stroke),

\footnotetext{
* To whom all correspondence should be addressed. E-mail: predeepphdcse@gmail.com
}

Metabolic diseases (e.g. diabetes, parathyroid, pituitary, adrenal), Musculoskeletal Disabilities (e.g. arms, legs, spine, paraplegia, quadraplegia), Psychiatric disorders (e.g. mental, emotional, personality, psychotic), effects of drugs (e.g. sedatives, tranquillizers, antidepressants, narcotics), effects of Alcohol (e.g. alcohol dependency) and aging Issues (e.g. loss of strength, slow reaction time, lack of attention, confusion, progressive dementia $)^{19}$. Among these issues, fatigue and sudden heart attack are the major causes of accidents which cannot be controlled by driver even if he/she wants to do.

The post-operative patients can develop complications once they are discharged from hospital. Hence the ECG of such patients needs to be monitored for some time after their treatment. 
This helps in spotting the improper working of the heart and take safety measures. Certain of these lives can often be saved if desperate care and cardiac treatment is delivered within the so-called golden hour. So the need for guidance on first hand medical attention becomes inexorable. Hence, patients who are at risk require that their cardiac health to be monitored recurrently whether they are indoors or outdoors so that emergency cure is possible. So this device determines a fatigue through a simple three step facial image processing algorithm using a web camera. For determing myocardial abnormalities the ECG of the driving person is acquired from the ECG sensors wrapped onto the steering wheel. Once changes detected the Microconroller unit will send an alert message to the medical practitioner through GSM.

\section{Previous research}

There are several techniques exist for this drowsy behaviour detection. The analysis of drowsy driving behaviour by means of performing face detection is the basic step for eye detection. In which, eyes are detected by constructing a threshold on roundness of iris and analyses the eye state for drowsiness detection ${ }^{16}$. Several techniques exist, which detect fatigue based on the fuzzy fusion of blinking features that are extracted from a high frame rate video ${ }^{15}$. Another detection algorithm which proceeds from face detection performs eye detection and calculates eye blink pattern from "Eye blink pattern detection algorithm" ${ }^{3}$. Illumination based approaches also exist which uses an infrared image that remained stable regardless of whether it was used during the day or at night ${ }^{14}$. IR illumination cameras are used to detect the drowsy behaviour by means of dark pupil effect. Capturing Video motion and then converting into frames is a time consuming process where measuring the distances between the intensity changes in the eye area one can determine whether the eyes are open or closed ${ }^{2}$.

Even though these techniques are constructive, algorithms are little difficult to perform and of high execution time and cost. It is critical that several sensors are integrated and synchronized for a more realistic evaluation of the driver behaviour but the cost of the model design is too high. Determination of EEG signals is obtained by sensors which are placed on the head. Head movement of the driver is detected by placing inclinometer on the forehead. The physiological signal-acquisition module and embedded signalprocessing module were designed for long-term EEG monitoring from which the state of drowsiness is detected ${ }^{1}$. Few approaches used a variance map for the real-time detection of eye blinks. But the performance of this method strictly hinge on head movements.

The different constraints using other methods are described in Table 1 . Our second objective is to monitor the abnormal ECG of the person driving. A wireless ECG device equipped with a built-in automatic warning expert system was developed where acquired ECG signals are instantaneously transmitted to mobile devices, such as netbooks or mobile phones through Bluetooth, and then, processed by the expert system $^{11}$. Most of the systems require an internet server for data transmission to the medical practitioner. Bluetooth ECG transmission is only applicable for a shorter distance.

In our proposed method the detection of QRS complexes in an Electrocardiogram (ECG) signal provides information about the heart rate within the heart as well as various abnormalities such as hyperkalemia, cardiac hypertrophy, etc. So we have analyzed different algorithms for detecting abnormal ECG and developed a prototype model that detects heart rate form ECG sensors wrapped onto steering wheel from which it calculates the time interval for the driver's pulses and converts the time interval into a pulse rate. If any unusual signs detected then the programmed Microcontroller will sent an alert SMS to the medical practitioner through GSM.

\section{MATERIALSAND METHODS}

The method of analyzing fatigue through image obtained from continuously running video input is shown in the figure.1. Initial step starts with the acquisition of an image from the camera followed by face tracking and eye detection. If eyes are open then it checks the heart rate of the driving person. If the heart rate is lesser than the predefined threshold then the person is subjected to feel drowsiness. At the other end usually the Biosignals are acquired from sensors. That converts the bio signal into an electrical signal to the system. If the heart rate is higher than the threshold it is 
classified as an attack and an alarm is triggered. The experimental setup is discussed in detail below.

\section{Face Detection}

Face detection is performed as the essential step for eye localization. Face detection is done by means of local SMQT features and split up SNoW classifier. The first step is preprocessing. It is used to calculate the integral images and lighting corrections from the scaled (scale factor is 1.2) original image. The second step is processing the frame sequences along with trained threshold values. The third step is postprocessing. It clusters the adjacent detected faces to one or several rectangles to represent faces according to distance factor.

At first the feature window $(16 \times 16)$ is fixed; we need to scale the source images down to detect faces which originally are large in size. The down-sampling uses simple linear image interpolation technique with a factor of 1.2. The most important thing during this step is to calculate the integral images and the lighting corrections. After making integral images, this step is performed to find whether face pixel exist in image or not. This can be done by comparing it with defined threshold values. After processing with threshold values operated onto the original source image and all scaled images, we cluster the detected face pixels for any adjacent scaled images to be the final detected face rectangle.

\section{Eye detection}

After detecting the face, the location of the eyes will be detected using the duration of eye closure, Frequency of eye blinks. The location of the eye is detected by RGB to grey conversion, binary image conversion, and number of connected components is analysed. Normally duration of eye closure is 0.15 to 0.25 seconds. By measuring duration of eyelid closure, the abnormal blink rate is measured and drowsy driving behaviour was analysed. Low Eye blink frequency can also be brought into account in drowsiness detection. If the eyes are found closed for a certain threshold then immediately the system detects drowsiness and provides an alarm to the person. If open it proceeds to heart rate detection.

\section{ECG based Heart rate variability analysis}

Heart rate can vary as the body's need to absorb oxygen and excrete carbon dioxide changes, such as during exercise or sleep. ECG signal consists of six segments P-Wave, P-R Interval, QRS Complex, Q-T Interval, T Wave, and S-T Segment. This paper mainly concentrates on R-R interval which itself provides enough details about drowsy behaviour. It is easy to pick up heart rate with IR sensors but we know HR varies significantly during exercise, running and even when the person is subjected with stress. So we have acquired heart rate from ECG. Our system uses a non-invasive method of picking up an ECG through ECG sensors wrapped with electrically conductive fabric (ECF) as two ECG electrodes.

\section{Signal Conditioning}

ECG signals acquired are very small electrical signals in the incidence of higher noise components. The electrical signal is very weak (normally 0.0001 to 0.003 volt) in amplitude. These signals are within the frequency range of 0.05 to $100 \mathrm{~Hz}$. If they are not amplified a greater gain is needed to convert the weak differential signals into characteristic signals, causing the circuit to douse easily. So here the signal conditioning includes both amplifying and filtering.

\section{ECG Noise Filtering}

Once after getting the ECG signal the next step is to remove the noise associated with the signal and extract the noise free signal. Baseline wandering, or inessential low-frequency highbandwidth components, can be caused by respiration and body movements. It can cause problems to analysis, exclusively when inspecting the low-frequency ST-T segment.

The cut-off frequency should be selected so as to ECG signal information remains unembellished while as much as possible of the baseline wandering is removed and so the lowestfrequency component of the ECG should be acquired. Power line interference from power lines can cause 50/60 Hz sinusoidal interference, possibly essorted by some of its harmonics. Such noise can cause complications interpreting lowamplitude waveforms and spurious waveforms can be pioneered. Different types of filters for obtaining a noise free ECG signal are shown in Table.3.

\section{$R$ peak detection}

After noise removing process, the ECG signal is extracted. The PQ interval (also known as the PR interval) is the amount of time from the beginning of the P complex to the QRS complex. This signifies the extent of time between the 
commencement of atrial contraction and the start of ventricular contraction. Finding out the normal QRS-complex from the P- and T-waves does not make complications because it has a specific waveform and overlooking amplitude. This amplitude is nearby $1 \mathrm{mV}$ in a normal heart and the normal time of the QRS is $0.08-0.10 \mathrm{~s}$. A sinus rhythm greater than $100 / \mathrm{min}$ is called sinus tachycardia and a sinus rhythm lesser than $60 / \mathrm{min}$ is called sinus bradycardia. It occurs from congestive heart failure. Prolonged QRS will result in Second order attack. So our system focuses on QRS complex wave and $\mathrm{RR}$ interval since QRS complex wave is the most central, visual part and less affected by noise.

Our proposed work is concentrating only on RR intervals where RR interval is the time between QRS complexes. By processing this R-R interval, the decrease or increase in $\mathrm{LF} / \mathrm{HF}$ frequency ratio while driving is analysed and processed to the microcontroller to provide an alert SMS to the medical practitioner.

\section{ARM Microcontroller}

The various modules in the system are integrated and controlled by ARM7 microcontroller. It has support for modem control with maximum UART data bit rate of 4.5Mbps and built in ADC and PWM ports. After determining the average heart rate, decision is taken based on our threshold $(60<$ Heart rate $>100)$ to determine abnormal heart rate. GSM modem is interfaced with controller through AT commands and will send an alert message to the patient and medical practitioner at a same time.

\section{RESULTSAND DISCUSSION}

Once the image acquisition has been completed our model detects the face by calculating centroids. The correlation coefficient was compared with the threshold and drowsiness was detected. The eye opening and eye closure time were calculated and depending upon the blinks the results indicates that eyes are closed and drowsiness detected.

\section{Simulation results for ECG Signal Processing}

We have performed an analysis using LabVIEW by taking a noisy ECG signal and determined $\mathrm{R}$ peaks from it. The ECG simulator endows us to scrutinize normal and abnormal ECG waveforms without essentially using the ECG sensors and DAQ board. The insinuation is to apply direct fast Fourier transform (FFT), remove

Table 1. Bottlenecks of other fatigue detection systems

\begin{tabular}{|c|c|}
\hline Existing research work & Limitations \\
\hline Iris recognition [3] & $\begin{array}{l}\text { Iris obstruction by eyebrows, eyelashes, eyelids, Lightning reflections, } \\
\text { specular reflections, Poor focus, partially captured iris, Out-of image iris, } \\
\text { and Off-angle iris motion may occur. }\end{array}$ \\
\hline Fuzzy and neural networks [7] & $\begin{array}{l}\text { Needs a huge training set of face and non-face images and take a long time in } \\
\text { execution. }\end{array}$ \\
\hline $\begin{array}{l}\text { Eye blink pattern detection } \\
\text { algorithm [8] }\end{array}$ & $\begin{array}{l}\text { The presence of glasses may affect the core components of the system } \\
\text { including face detection, eye detection. }\end{array}$ \\
\hline Illumination based approaches [9] & $\begin{array}{l}\text { Although IR based approaches perform reasonably at night time it requires } \\
\text { the necessity of installing an IR LEDs setup and maintenance. }\end{array}$ \\
\hline Capturing video motion [12] & Converting into frames increases the computation complexity \\
\hline $\begin{array}{l}\text { Long-term EEG monitoring } \\
{[4],[13,[16]}\end{array}$ & $\begin{array}{l}\text { If we use EEG, it will make more physical disturbances to driver, stress } \\
\text { signals will also mix. }\end{array}$ \\
\hline
\end{tabular}

Table 2. Approaches for detecting abnormal myocardial behaviour

\begin{tabular}{ll}
\hline ECG monitoring & Limitations \\
\hline Tele-cardiology system [20] & $\begin{array}{l}\text { An alert signal is sent to the remote database server, which can be } \\
\text { accessed by an Internet browser, once an abnormal ECG is detected. } \\
\text { Too expensive for real time modelling }\end{array}$ \\
Driver Alertness Monitoring system [14]
\end{tabular}


Table 3. Methods used for noise free ECG signal extraction

\begin{tabular}{ll}
\hline Different methods of filtering & Types of noise removal \\
\hline Low pass filter and High pass filter & Drift due to respiration \\
8 point moving average filter and Notch filter & Power line interference removal \\
Recursive least square adaptive notch filter & Baseline drift removal \\
FIR high pass filter & Baseline wandering \\
Adaptive filter & Muscle noise removal \\
Band pass filter & Low amplitude noise at output \\
Wiener filter & High frequency noises of ECG \\
FIR equiripple band pass and FIR butter worth filter & Composite noises \\
\hline
\end{tabular}

Table 4. Test results obtained from HRV analysis

\begin{tabular}{lcc}
\hline S.No & Average Heart rate(BPM) & Symptoms \\
\hline 1 & 62 & Normal \\
2 & 72 & Normal \\
3 & 115 & Sinus Tachycardia \\
4 & 55 & Sinus Bradycardia \\
5 & 117 & Sinus Tachycardia \\
6 & 45 & Sinus Bradycardia \\
7 & 78 & Normal \\
8 & 66 & Normal \\
9 & 44 & Sinus Bradycardia \\
\hline
\end{tabular}

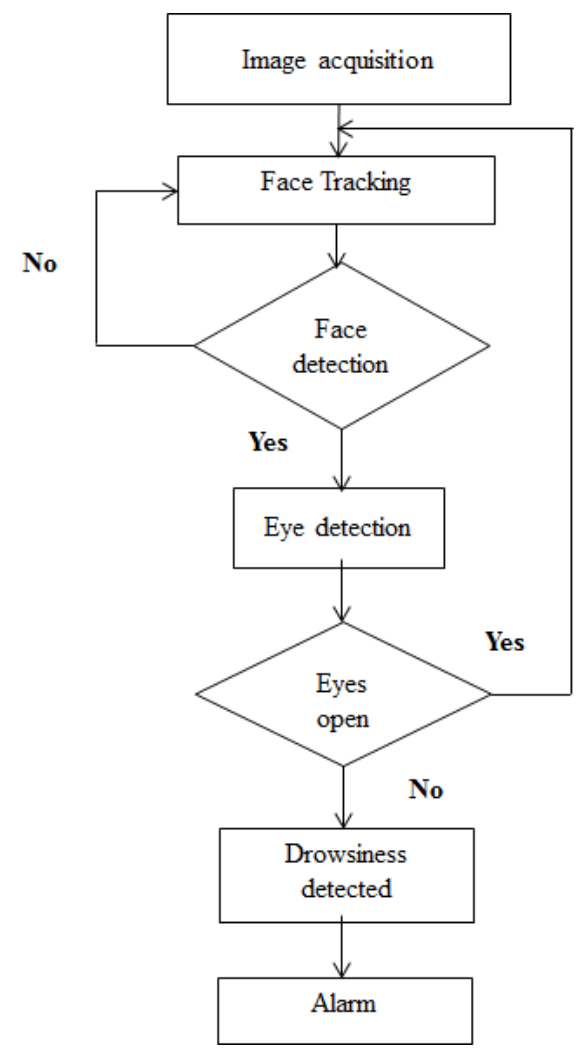

Fig. 1. Facial Image processing for drowsiness detection low frequencies and restore ECG as shown in figure 6. Band pass filter was applied to the input ECG and noise free ECG was obtained.

LabVIEW Biomedical Toolkit affords a Bio signal filtering, file which we can design a Kaiser Window FIR high pass filter to remove the baseline wandering. Besides to digital filters, the wavelet transform is also an effective way to remove signals within precise sub bands. As shown here in figure 8 we have used the Daubechies2 (db02) wavelet because this wavelet is alike to the real ECG signal. Here, depending upon the sampling duration and sampling points of ECG signal, the trend level is set. It is calculated as shown below

Trend level $=\{\log 22 \mathrm{t} / \log 2 \mathrm{~N}\}$

Where $t$ is the sampling duration and $\mathrm{N}$ is the number of sampling points. After baseline wandering is removed, the resultant ECG signal is more immobile than the original signal. However, some other types of noise discussed in table 1 might still affect feature extraction of the ECG signal. The noise may be complex stochastic processes within a wideband, so it is not possible to remove those by digital filters. To remove the wideband noises, we have used the Wavelet Denoise Express VI and the results are shown in figure 6. The next step is to predict QRS and RR intervals since heart rate is calculated from successive RR intervals. The results are shown in of figure 6 .

By applying peak detection heart rate is calculated. The statistics taken from the above results are shown in the below table 4 . Subjects with a heart rate lesser than 60 are prolonged to affect with sinus Bradycardia and have experienced drowsy feeling. 


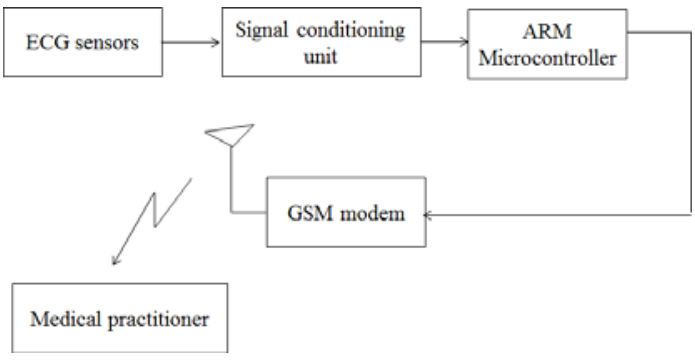

Fig. 2. Proposed Tele - cardiac driver alert system

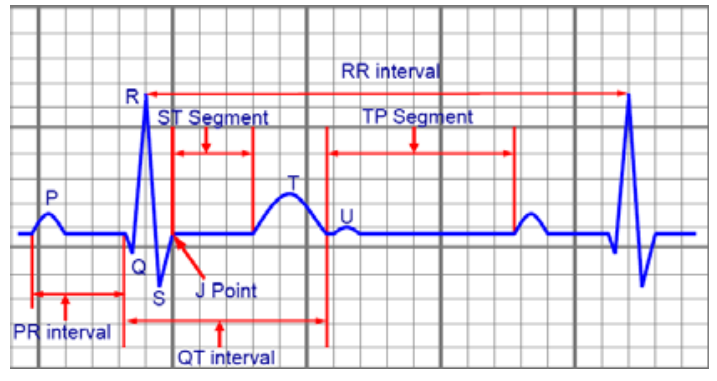

Fig. 3. Typical ECG waveform

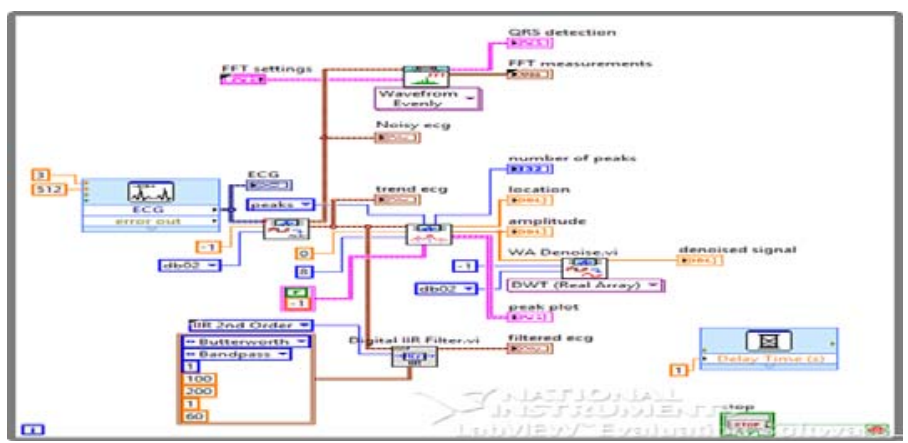

Fig. 4. LabVIEW design for ECG signal processing

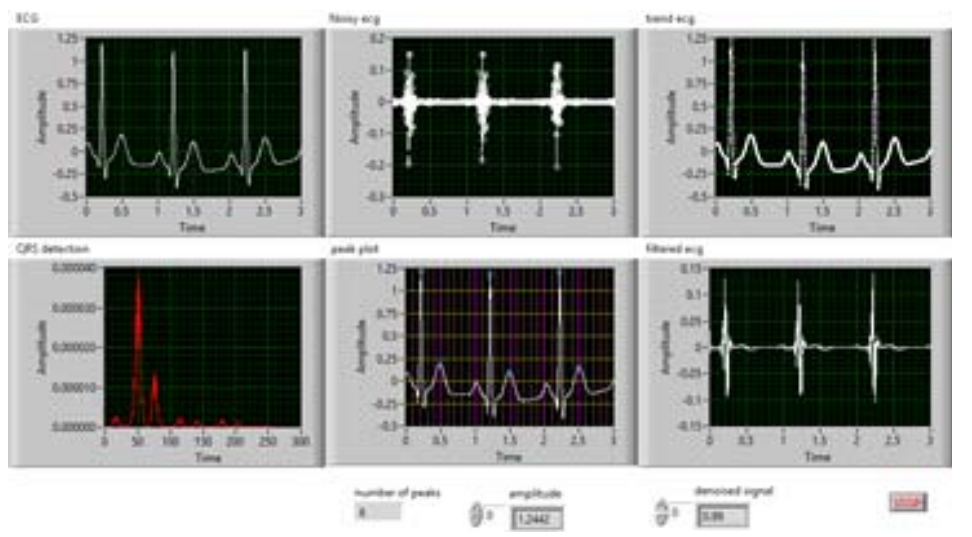

Fig. 6. Output results with QRS complex detection

\section{CONCLUSION}

This research work provides a collective analysis for determining the perilous activity of person in driving state. We have analysed the behavioural changes of a person in driving state by combining the two major parameters eye state and heart rate variability. In this first part of research work we have examined the driver's state of drowsiness on-line, which requires low computational and time complexity. We tried to face this constraint by using unfussy image processing techniques by means of a simple detection algorithm via Matlab as a tool. Use of normal web camera, fast computation obtained from scaling factor compromise the systems performance for the sake of simplicity which is a major criterion for real time scenario. This proposed model is very cost effective i.e it can be implemented with mobile cameras which now-a-days have high pixel quality. This algorithm even works for candidates wearing spectacles and in lighting environment up to an extent. 


\section{REFERENCES}

1. Chien-zhi ou, Chiao-tung univ, Hsinchu etal (2012) Brain Computer Interface-based Smart Environmental Control System. In Proceedings of IEEE Eighth International Conference on Intelligent Information Hiding and Multimedia Signal Processing (IIH-MSP), 281-284.

2 .Devi R, Sarada M, Preeti R et al., Driver Fatigue Detection Based on Eye Tracking. In Proceedings of First International Conference on Emerging Trends in Engineering and Technology, 2008; 649 -652.

3. Danisman T, Bilasco IM, Djeraba C et al., Drowsy Driver Detection System Using Eye Blink Patterns. In proceedings of IEEE International conference on machine and web intelligence, 2010; 230-233.

4. Caffier P, Erdmann U, Ullsperger.P., Experimental evaluation of eyeblink parameters as a drowsiness measure”. European Journal of Applied Physiology, 2003; 89:319-325..

5. Kima YS, Baekb HJ, etal., "Helmet based physiological Monitoring System”, European Journal of Applied Physiology, 2009; 365-372.

6. Lee BG, Jung SJ, Chung WY., Real-time physiological and vision monitoring of vehicle driver for non-intrusive drowsiness detection. IET Journal of Communications, 2011; 2461 2469.

7. Lee, Boon-Giin, Chung, Wan-Young., Driver Alertness Monitoring Using Fusion of Facial Features and Bio-Signals. IEEE Sensors Journal, 2012; 2416-2422.

8. Lin, Chin-Teng, Li-Wei et al., Adaptive EEGBased Alertness Estimation System by Using ICA-Based Fuzzy Neural Networks. IEEE Transactions on Circuits and Systems I , 2006; 2469 - 2476.

9. Lin, Chin-Teng, Chang et al., A Real-Time Wireless Brain-Computer Interface System for Drowsiness Detection. IEEE Transactions on Biomedical Circuits and Systems, 2010; 214222.

10. Lin Y, Leng H, Yang G et al., An Intelligent
Noninvasive Sensor for Driver Pulse Wave Measurement, IEEE Sensors Journal, 2007; 790-799.

11. Lin CT, Cheng KC et al., An Intelligent Telecardiology System Using a Wearable and Wireless ECG to Detect Atrial Fibrillation. IEEE Transactions on Information Technology in Biomedicine, 2010; 726-733.

12. Mingheng Zhang, Linhui L et al., Study on vision monitoring techniques of driver's face orientation. In Proceedings of IEEE International Conference on Intelligent Control and Information Processing, 2010; 297-301.

13. Morris T, Blenkhorn P, Zaidi F., Blink detection for real-time eye tracking. Journal of Network and Computer Applications, 2002; 129-143.

14. Park I, Hyeran B et al., Efficient Measurement of Eye Blinking Under Various Illumination Conditions for Drowsiness Detection Systems. In Proceedings of IEEE $18^{\text {th }}$ International conference on Pattern recognition, 383-386, 2006.

15. Picot A, Charbonnier S, Caplier A., Drowsiness detection based on visual signs: blinking analysis based on high frame rate video”, In Proceedings of IEEE International Instrumentation and Measurement Technology Conference (I2MTC'10), 2010.

16. Seifoory H, Taherkhani D, Arzhang B et al., An Accurate Morphological Drowsy Detection. In International Proceedings of Computer Science and Technology, 2011; 51-54.

17. Yang Y, Mcdonald M, Zheng P., Can drivers' eye movements be used to monitor their performance? a case study. IET journal of Intelligent Transport Systems, 2012; 444 -452.

18. Xingliang Xion ,Lifang Deng, Yan Zhang et al., Objective Evaluation of Driver Fatigue by Using Spontaneous Pupillary Fluctuation. In proceedings of IEEE 5th International Conference on Bioinformatics and Biomedical Engineering, 2011; 1-4.

19. Alberta Transportation Annual Report., http:// www.transportation.alberta.ca/Content/ docType47/Production/drvfitness.pdf, 2012. 